\title{
Resected Pleomorphic Carcinoma of the Gallbladder
}

\author{
Masanari Shimada ${ }^{a}$ Shinichi Kadoya ${ }^{a}$ Yasuko Tanada $^{a}$ \\ Naoki Endo ${ }^{a}$ Nozomu Murakami ${ }^{a}$ Kazuhiro Matsui ${ }^{b}$ \\ Departments of ${ }^{\mathrm{a}}$ Surgery and ${ }^{\mathrm{b}}$ Pathology, Saiseikai Takaoka Hospital, \\ Takaoka City, Japan
}

\section{Key Words}

Gallbladder · Pleomorphic carcinoma Squamous cell carcinoma Spindle cell . WHO classification

\begin{abstract}
Pleomorphic carcinoma is a rare lesion and the literature contains few reports of pleomorphic carcinoma of the gallbladder. The present study reports a rare case of primary pleomorphic carcinoma of the gallbladder for which we were able to perform curative surgery. A 77-year-old woman with dementia developed nausea and anorexia, and computed tomography demonstrated irregular thickening of the gallbladder wall. Drip infusion cholangiography and endoscopic retrograde cholangiopancreatography revealed no stenosis of the common and intrahepatic bile ducts. We suspected carcinoma of the gallbladder without lymph node metastasis and invasion to the common bile duct. We guessed it to be resectable and performed open laparotomy. At operation, the fundus of the gallbladder was adherent to the transverse colon, but no lymph node and distant metastases were detected. Therefore, we performed curative cholecystectomy with partial colectomy. Histopathology and immunostaining showed coexistence of an adenocarcinoma, squamous cell carcinoma and sarcomatous tumor of spindle-shaped cell, as well as transition zones between these tumors. We diagnosed stage I pleomorphic carcinoma of the gallbladder. No recurrence has been observed for one and a half years. The biological behavior of pleomorphic carcinoma of the gallbladder remains unknown. It will be necessary to accumulate more case reports of this tumor in order to define diagnostic criteria.
\end{abstract}

\section{Introduction}

The present study reports a rare case of primary pleomorphic carcinoma of the gallbladder for which we were able to perform curative surgery and identify long survival. Pleomorphic carcinoma is a rare lesion newly defined in the new World Health Organization (WHO) classification of lung tumor [1]. However, according to General 
Rules for Surgical and Pathological Studies on Cancer of the Biliary Tract [2], the tumor is not clearly described in the rules. The literature contains few reports of pleomorphic carcinoma of the gallbladder, hence there is no consensus or standard terminology regarding this tumor. Moreover, no guidelines for its diagnosis or treatment have yet been drawn up. Also, their reports showed few long survival cases [3, 4]. In their minimal reports, pleomorphic carcinoma of the gallbladder is classified into a subtype of undifferentiated carcinoma. Undifferentiated carcinoma may be classified into three types: (a) a small cell type, composed of fairly uniform small cells resembling classic oat cell carcinoma of the lung, (b) a spindle cell type, formed by spindle-shaped cells, presenting a sarcoma-like appearance, and (c) a pleomorphic type, characterized by a striking degree of pleomorphism of neoplastic cells and by a prominent inflammatory infiltrate [3]. Yet the present case showed unprecedented histopathological findings that contained all components of squamous cell carcinoma, adenocarcinoma and sarcoma, so we would like to suggest new histological knowledge about carcinoma of the gallbladder.

\section{Case Report}

A 77-year-old woman with dementia presented with nausea and anorexia lasting for a few days and was admitted to our hospital. Physical examination upon admission was unremarkable. Laboratory investigations showed mild hepatobiliary inflammation and mild elevation of serum tumor marker levels: white blood cell count 8,100/ $\mu$ l, C-reactive protein $8.19 \mathrm{mg} / \mathrm{dl}$, aspartate aminotransferase $43 \mathrm{IU} / \mathrm{l}$, alanine aminotransferase $105 \mathrm{IU} / \mathrm{l}$, alkaline phosphatase $659 \mathrm{IU} / \mathrm{l}$, $\gamma$-glutamyl transpeptidase $148 \mathrm{IU} / \mathrm{l}$, total bilirubin $0.68 \mathrm{mg} / \mathrm{dl}$, amylase $34 \mathrm{mg} / \mathrm{dl}$, carcinoembryonic antigen $1.2 \mathrm{ng} / \mathrm{ml}$, carbohydrate antigen $19-962.8 \mathrm{U} / \mathrm{ml}$, squamous cell carcinoma-related antigen $0.7 \mathrm{ng} / \mathrm{ml}$, hepatitis B virus surface antigen and hepatitis C virus antibodies negative.

Abdominal computed tomography and ultrasonography showed irregular thickening of the gallbladder wall (fig. 1a, b). We therefore suspected a neoplasm of the gallbladder and direct invasion of the adjacent liver parenchyma. Drip infusion cholangiography and endoscopic retrograde cholangiopancreatography revealed no stenosis of the common and intrahepatic bile ducts. The part of the origin of the cystic duct was visible, and we therefore assumed the tumor had not invaded the common bile duct (fig. 1c, d).

Based on these preoperative imaging findings, carcinoma of the gallbladder was diagnosed, and we performed open laparotomy. At operation, the fundus of the gallbladder was tightly adherent to the transverse colon, but although examination of frozen section revealed proliferation of atypical cells in the body of the gallbladder, there was no invasion of the gallbladder bed or the cystic duct and no metastasis to the lymph nodes along the cystic duct. Therefore, we were able to perform curative cholecystectomy with partial colectomy ( $\underline{\text { fig. } 2}$ ). The patient's postoperative course was uneventful. Although her appetite failed to improve because of the dementia, she could be discharged on postoperative day 43 .

The resected lesion appeared to be two fused tumors: one protruding glanular lesion at the fundus (lesion A) and one larger protruding lesion at the body (lesion B). Lesion A measured $3.5 \times 1.0 \mathrm{~cm}$, and lesion B measured $9.0 \times 9.0 \mathrm{~cm}$. The resected transverse colon contained a perforation between the fundus of the gallbladder and the mucosa of the colon (fig. 3 ). Histopathological examination and immunostaining showed coexistence of an adenocarcinoma, squamous cell carcinoma and sarcomatous tumor of spindle-shaped cells as well as areas of transition between these lesions ( $\underline{\text { fig. } 4}$, fig. 5). The tumor reached the muscularis propria but did not extend extramurally. Lesion A was a well-differentiated tubular adenocarcinoma immunohistologically positive for cytokeratin 7 (CK7) and CK18. Lesion B was a predominantly squamous cell carcinoma with sarcomatous differentiation and was immunohistologically positive for CK5, CK6 and vimentin. Furthermore, lesion B contained areas that were positive for CK7, and it had a transitional zone of staining. Therefore, lesion B was suspected to arise from the fundal adenocarcinoma (lesion A), and 
we diagnosed 'pleomorphic carcinoma'. On the other hand, we confirmed that the colonic perforation was an ulcerative lesion only and that the tumor did not invade the colon.

The final pathological stage was stage I according to the General Rules for Surgical and Pathological Studies on Cancer of the Biliary Tract [2]. No recurrence has been observed for one and a half years.

\section{Discussion}

In 1998 the third edition of the WHO classification 'Pathology and Genetics of Tumors of the Lung, Pleura, Thymus and Heart' proposed the independent entity of 'carcinoma with pleomorphic, sarcomatoid or sarcomatous elements'. The criteria for classification as pleomorphic carcinoma are as follows: (1) histologically detected sarcomatoid lesion composed of spindle cells and giant cells within a tumor such as squamous cell carcinoma, adenocarcinoma or large cell carcinoma, (2) sarcomatous components accounting for more than $10 \%$ of the tumor, and (3) the tumor being composed only of spindle cells and giant cells [1].

Until the present report, pleomorphic carcinoma of the gallbladder has been reported as a pleomorphic type of undifferentiated carcinoma proposed by Guo and Enjoji [3]. It is characterized by confirming pleomorphic giant cells, bizarre cell with single or multiple atypical large nuclei, and the characteristic findings of tumor cells having phagocytosed each other (cannibalism) [3-6].

The histopathological features of the present case were as follows: (1) coexistence of adenocarcinoma and squamous cell carcinoma, both of which were positive for cytokeratins, (2) transitional zones between the tumors, and (3) positivity for vimentin indicating dedifferentiation. Accordingly, although our case is quite similar to the 'so-called carcinosarcoma of the gallbladder', which consists predominantly of squamous cell carcinoma, we made a diagnosis of pleomorphic carcinoma because of the mixture of adenocarcinoma, squamous cell carcinoma and sarcomatous tumor arising from the fundal adenocarcinoma. Since the present case contained no pleomorphic cell, it was thought to differ from the pleomorphic carcinoma proposed by Guo and Enjoji [3]. On the other hand, our case did not have heterotopic elements such as malignant bone, cartilage or other mesenchymal tissues, which were useful findings to differentiate carcinosarcoma from pleomorphic carcinoma. Therefore, we could confirm that the present tumor was pleomorphic carcinoma. Since the present case met the diagnostic criteria for pleomorphic carcinoma of the lung, we propose that similar tumors should be classified as pleomorphic carcinoma of the gallbladder.

The hypothesis of the origin of carcinosarcoma is very helpful when considering the reason for coexistence of epithelial tumor and sarcomatous tumor. Recently, the most widely supported hypothesis is that the epithelial tumor undergoes metaplasia to become a sarcoma-like tumor [7]. Moreover, the hypothesis of metaplasia from adenocarcinoma to squamous cell carcinoma is the leading etiological theory behind coexistence of adenocarcinoma and squamous cell carcinoma, although consensus has not been reached on this issue [8].

With only a few reports of therapy for pleomorphic carcinoma of the gallbladder in the literature, the optimal curative approach is unclear. We assume that it would 
be similar to that for squamous cell carcinoma or carcinosarcoma. In other words, surgical resection with the aim of cure is regarded as the first choice, albeit with clinical staging, if there is no metastasis to other organs. Furthermore, there are few reports of the responsiveness of squamous cell carcinoma or carcinosarcoma to chemotherapy or radiation [9].

The present case consisted predominantly of squamous cell carcinoma. An $87.5 \%$ five-year survival has been reported when curative surgery is performed for this type of tumor, suggesting the potential for long survival [9].

In conclusion, the biological behavior of pleomorphic carcinoma of the gallbladder remains unknown. It will be necessary to accumulate more case reports of this tumor in order to define diagnostic criteria.
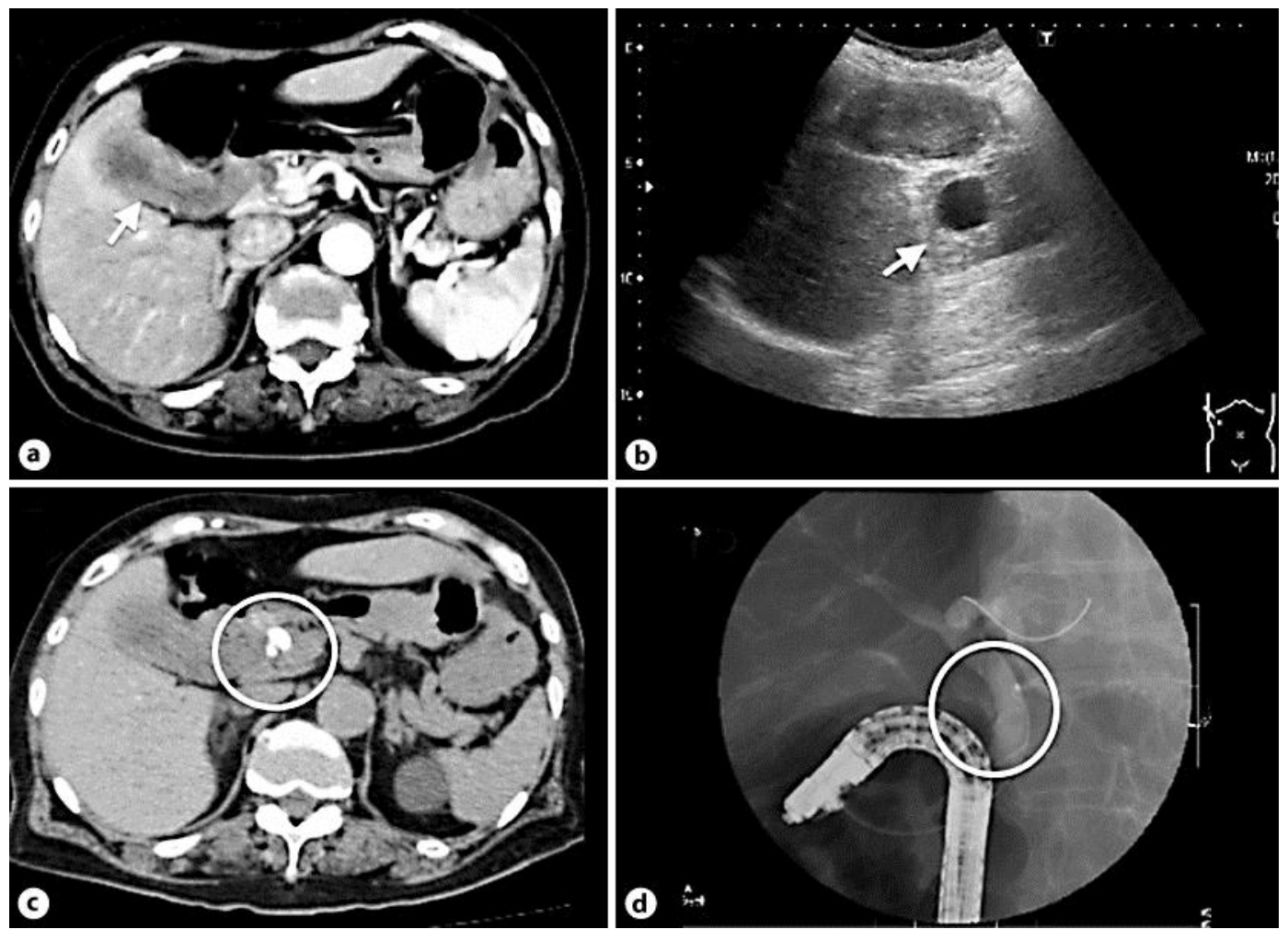

Fig. 1. Preoperative examinations. Abdominal computed tomography (a) and ultrasonography (b) showed irregular thickening of the gallbladder wall (arrows). Drip infusion cholangiography (c) and endoscopic retrograde cholangiopancreatography (d) showed no stenosis of the common and intrahepatic bile ducts (circles). 


\begin{tabular}{r|l|l|l}
$\begin{array}{r}\text { Case Reports in } \\
\text { Gastroenterology }\end{array}$ & $\begin{array}{l}\text { Case Rep Gastroenterol 2012;6:747-753 } \\
\text { DOI: 10.1159/000346295 }\end{array}$ & $\begin{array}{l}\text { Published online: } \\
\text { December 19, 2012 }\end{array}$ & $\begin{array}{l}\text { C 2012 S. Karger AG, Basel } \\
\text { ISSN 1662-0631 } \\
\text { www.karger.com/crg }\end{array}$ \\
\hline
\end{tabular}

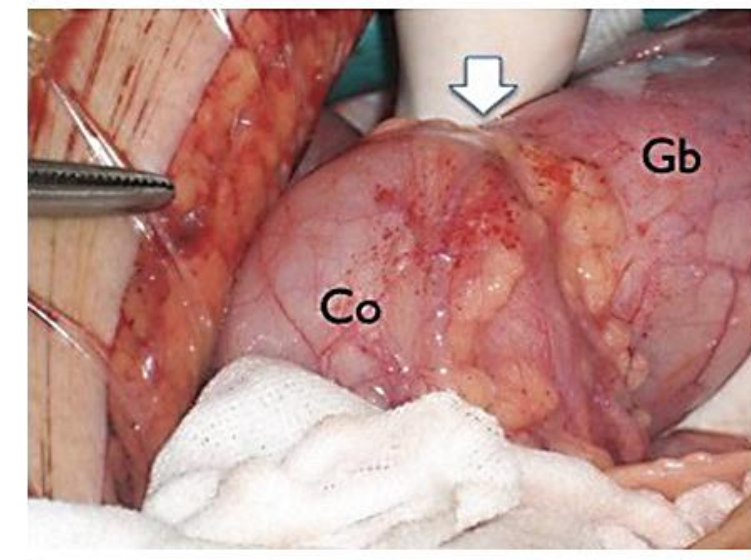

a

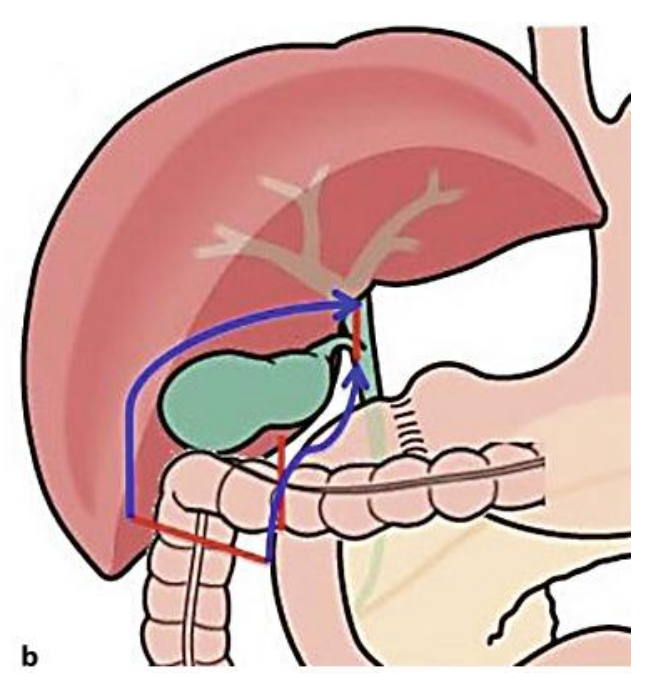

b

Fig. 2. Operative findings. The fundus of the gallbladder (Gb) was tightly adherent to the transverse colon (Co) (a, arrow). We performed curative cholecystectomy with partial colectomy (b).

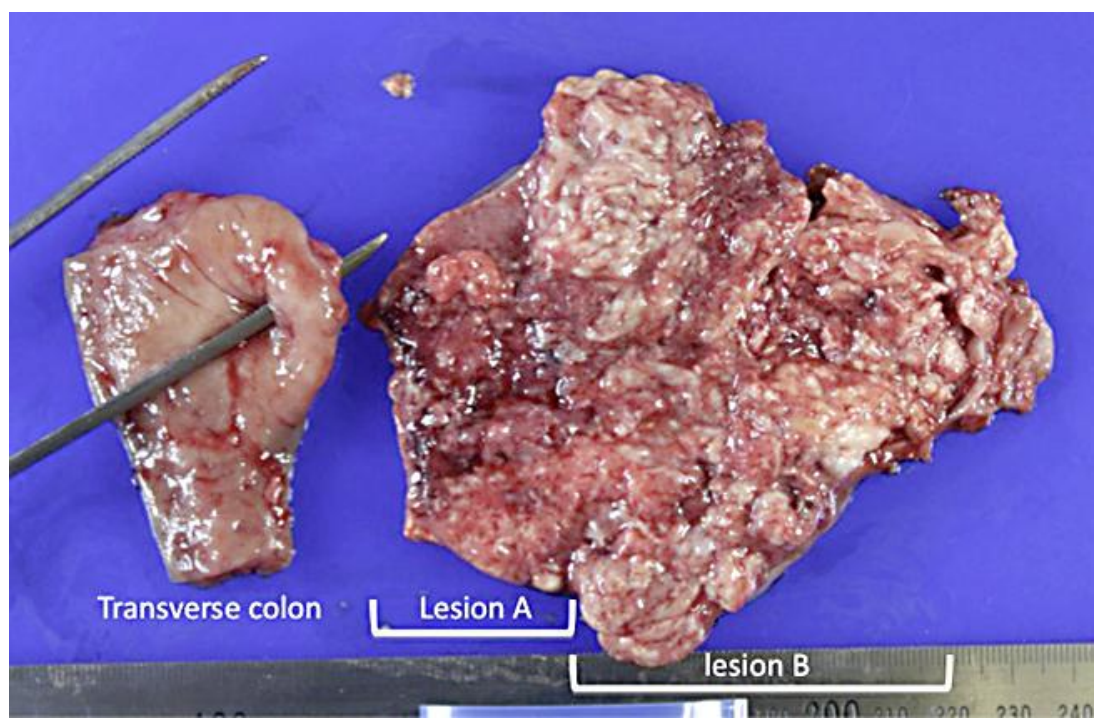

Fig. 3. Macroscopic features of the resected specimen. The resected lesion appeared to be two fused tumors: one protruding glanular lesion at the fundus (lesion $\mathrm{A}$ ) and one larger protruding lesion at the body (lesion B). Lesion A measured $3.5 \times 1.0 \mathrm{~cm}$, and lesion B measured $9.0 \times 9.0 \mathrm{~cm}$. The transverse colon contained a perforation between the fundus of the gallbladder and the mucosa of the colon. 


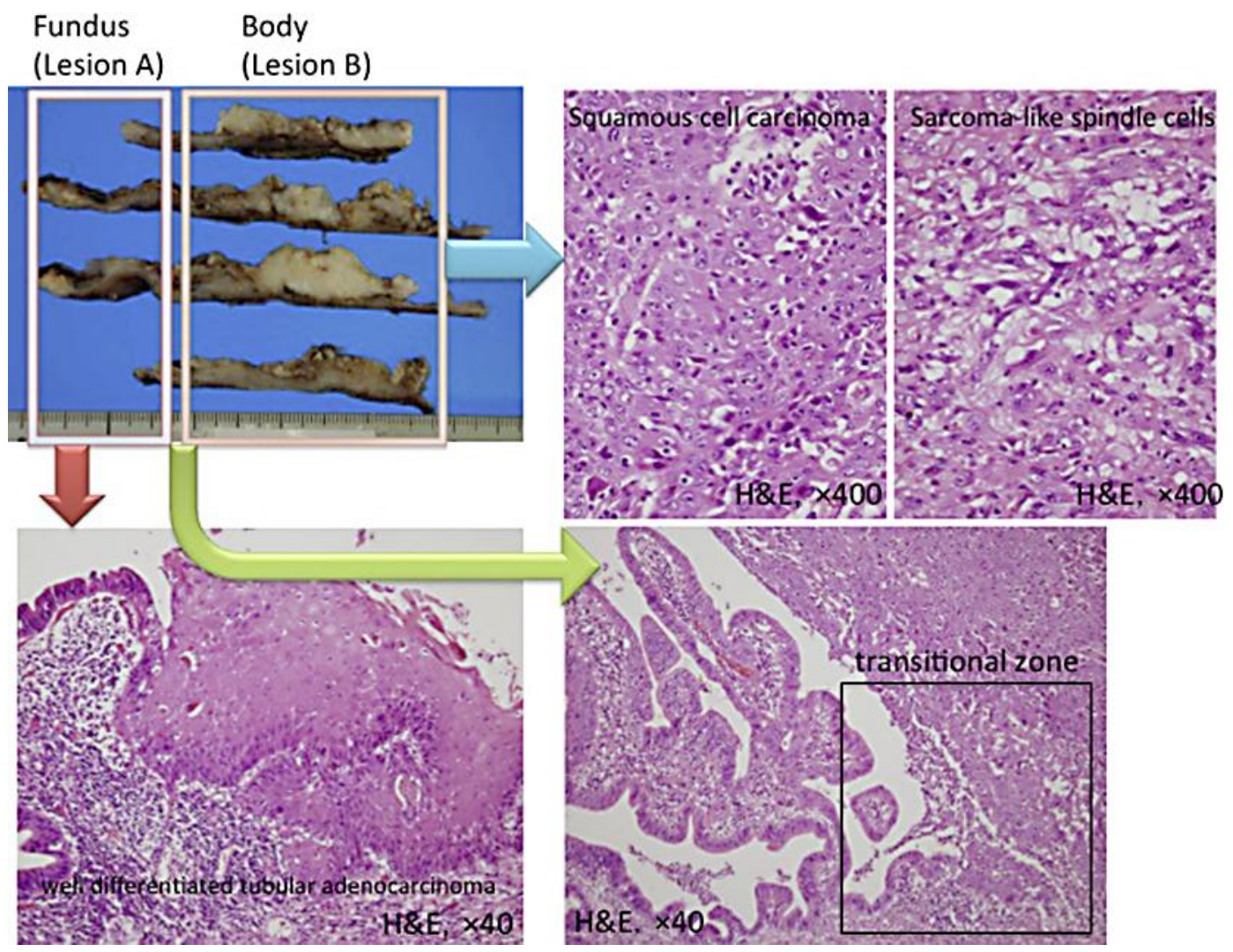

Fig. 4. Histopathological examination findings. H\&E staining showed coexistence of an adenocarcinoma, squamous cell carcinoma and sarcomatous tumor of spindle-shaped cells, as well as areas of transition between lesion A and lesion B (square). Lesion A was a well-differentiated tubular adenocarcinoma. Lesion B was predominantly squamous cell carcinoma with sarcomatous differentiation.
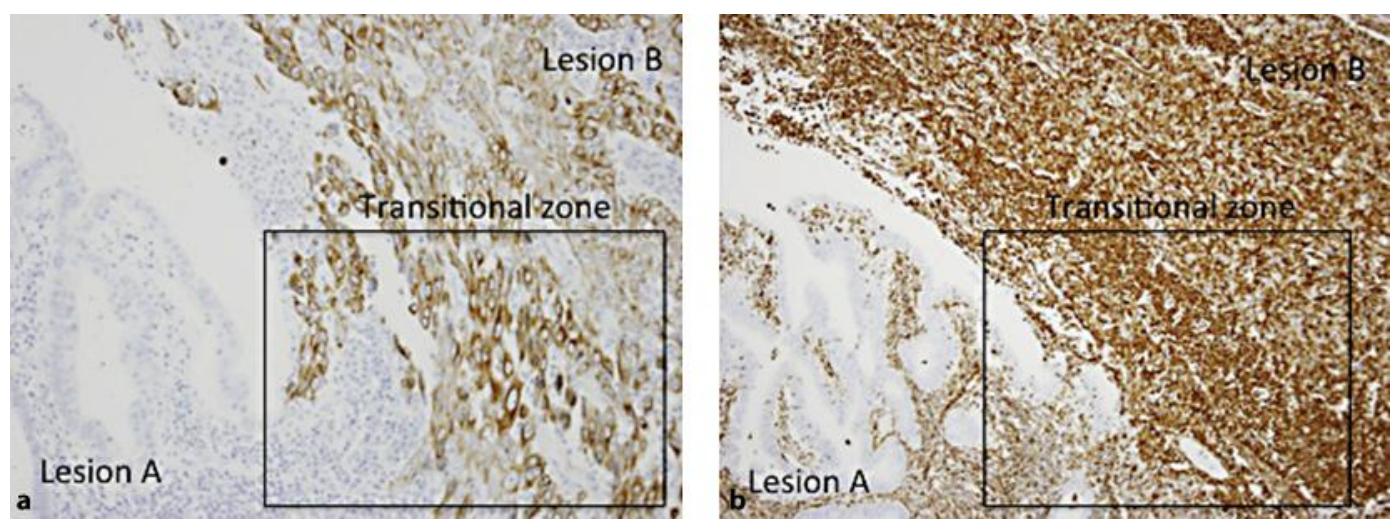

Fig. 5. Immunostaining findings. Lesion B was immunohistologically positive for CK5, CK6 (a) and vimentin (b), and a transitional zone of staining was found (squares). 


\section{References}

1 Brambilla E, Travis WD, Colby TV, Corrin B, Shimosato Y: The new World Health Organization classification of lung tumours. Eur Respir J 2001;18:1059-1068.

2 Japanese Society of Biliary Surgery: General Rules for Surgical and Pathological Studies on Cancer of the Biliary Tract. 2003.

3 Guo KJ, Enjoji M: Undifferentiated carcinoma of the gallbladder. Cancer 1988;61:1872-1879.

4 Caruso RA, Famulari C, Giuffré G, Mazzeo G: Pleomorphic carcinoma of the gallbladder: report of a case. Tumori 1991;77:523-526.

5 Kunimura T, Morohashi T, Kanda M: 4 cases of pleomorphic carcinoma of the gallbladder morphological and immunohistological study. Tandou 1993;7:188-194.

6 Maeda K, Sugawara H, Sasaki M, Matsui K, Tanada Y: A case of undifferentiated pleomorphic carcinoma of the gallbladder. Nihon Rinsyougeka Gakkai Zasshi 2007;68:2866-2871.

7 Ishimine T, Kameyama S, Isa T, Kohakura F, Koami H, Nagamine Y: A case of squamous cell carcinoma of gallbladder. Geka 2011;73:560-563.

8 Matsukiyo H, Watanabe M, Asai K, Osawa A, Nagao J, Sumiyama Y, Oharaseki T: A case of 'so-called carcinosarcoma of the gallbladder' associated with acute cholecystitis. Nihon Rinsyougeka Gakkai Zasshi 2009;70:1491-1496.

9 Horiguchi M, Iwabuchi M, Kawabata K: A resected case of squamous cell carcinoma of gallbladder diagnosed as bile duct carcinoma preoperatively and analysis of 28 resected cases from Japanese literatures since 1970 and questionnaire study. Tandou 1999;13:21-31. 